Series A

I. MATHEMATICA

434

\title{
ÜBER DAS \\ VERALLGEMEINERTE DIRICHLETPROBLEM FÜR LINEARE PARTIELLE DIFFERENTIALGLEICHUNGEN
}

VON

PETER HESS

H E L S I N K I 1969

S U O M A L A I N N TIEDEAKATEMIA

doi:10.5186/aasfm.1969.434 
Am 10. Nai 1968 vorgelegt von R. Nevanlinna und G. Järnefelt 


\section{Einleitung}

1. In der vorliegenden Arbeit untersuchen wir das verallgemeinerte Dirichletproblem für eine allgemeine (nicht notwendigerweise formal selbstadjungierte oder elliptische) lineare partielle Differentialgleichung.

2. Rolf Nevanlinna hat in Vorträgen 1952-1953 sowie in seinem Artikel [23] auf den engen Zusammenhang zwischen dem verallgemeinerten Dirichletproblem für eine (nichtelliptische) formal selbstadjungierte Differentialgleichung und dem Problem der Konstruktion einer Projektion auf einen Unterraum eines Hilbertraumes in bezug auf ein zweites "indefinites» Hermitesches Skalarprodukt hingewiesen. Als erste Anwendung der von Nevanlinna ([22] - [26]) entwickelten Theorie der indefiniten Skalarprodukte hat I. S. Louhivaara ([14] und [15]) Randwertprobleme für eine spezielle gleichmässig elliptische formal selbstadjungierte Differentialgleichung behandelt. Entsprechende Resultate für allgemeinere gleichmässig elliptische formal selbstadjungierte Differentialgleichungen wurden später mit ähnlichen Methoden von S. Hildebrandt [8] im Anschluss an gewisse Untersuchungen von E. Hölder erhalten. Louhivaara gab in [17] eine hinreichende Bedingung für die Existenz einer Lösung des verallgemeinerten Dirichletproblems für nichtelliptische formal selbstadjungierte Gleichungen; ein sowohl notwendiges als auch hinreichendes Kriterium für dasselbe Problem leitete dann F. E. Browder [4] her.

Die erste Veröffentlichung über nicht formal selbstadjungierte (nichtelliptische) Differentialgleichungen unter Verwendung verwandter Methoden stammt von W. Littman [13].

Diese Resultate bezogen sich alle auf Differentialgleichungen der Ordnung 2 oder $2 \mathrm{~m}$, die eine (in einem geeigneten Funktionenraum) beschränkte "Dirichletsche» Bilinearform erzeugen. In einer weiteren Arbeit [5] versuchte Browder, sich von diesen Einschränkungen zu befreien; er betrachtete nicht formal selbstadjungierte Gleichungen beliebiger Ordnung $r$.

Ju. P. Ginzburg und I. S. Iohvidov leiteten in ihrem Übersichtsartikel [7] eine neue Bedingung für die Existenz einer Projektion in bezug auf 
ein indefinites, nicht notwendigerweise Hermitesches Skalarprodukt her. Einer Idee von S. Hildebrandt und E. Wienholtz [9] folgend, kam S. Stenholm [28] zu ähnlichen Bedingungen.

Wegen einer Darstellung der Entwicklung der Nevanlinnaschen Theorie der indefiniten Skalarprodukte sei auf [19] verwiesen.

3. In den Kapiteln $1-3$ dieser Arbeit wird das verallgemeinerte Dirichletproblem für eine nicht notwendigerweise formal selbstadjungierte lineare partielle Differentialgleichung beliebiger Ordnung $r$ mit Randdaten ebenfalls beliebiger Ordnung $t$ behandelt. Das sehr allgemein formulierte verallgemeinerte Dirichletproblem (Problem I) wird in eine Fragestellung (Problem III) in dem Hilbertraum der Funktionen mit homogenen Randdaten umgeformt. Für die Existenz einer Lösung von Problem III wird notwendig und hinreichend sein, dass ein gewisses Element in der Wertemenge eines dicht definierten abgeschlossenen linearen Operators des betreffenden Hilbertraumes enthalten ist. In Kapitel 2 wird deswegen die Wertemenge eines linearen Operators charakterisiert; die dabei vorkommenden Resultate verfeinern Ergebnisse von Browder [4] und Stenholm [28]. In Kapitel 3 können wir dann ein notwendiges und hinreichendes Kriterium für die Lösbarkeit des verallgemeinerten Dirichletproblems angeben.

In Kapitel 4 werden die Resultate aus den früheren Kapiteln auf (nicht formal selbstadjungierte) gleichmässig stark elliptische Differentialgleichungen angewandt, wobei auch Differentialausdrücke mit unbeschränkten Koeffizienten zugelassen werden. Wir beweisen den bekannten Existenzsatz (den Fredholmschen Alternativsatz).

Die Frage, ob man mit dem allgemeinen Kriterium von Kapitel 3 noch andere konkrete Klassen von Differentialgleichungen behandeln kann, bleibt hier offen.

Die Anregung zu dieser Arbeit verdanke ich Herrn Professor Dr. I. S. Louhivaara. Für sein wohlwollendes Interesse und seine freundliche Hilfe bei der Abfassung des Manuskriptes sei ihm an dieser Stelle herzlichst gedankt. Ich möchte auch den Herren Professoren Dr. A. Huber und Dr. A. Pfluger für die wertvollen kritischen Bemerkungen beim Durchlesen der Arbeit meinen verbindlichsten Dank aussprechen. 


\section{Problemstellung und Definitionen}

1.1. Sei $\Omega$ ein beliebiges (offenes) Gebiet in einem $n$-dimensionalen reellen euklidischen Raum $R^{n}$. Für Funktionen $\varphi, \psi \in C^{t}(\Omega)$ $\left.(t=0,1, \ldots)^{1}\right)$ definieren wir das Skalarprodukt

$$
(\varphi, \psi)_{t}=\sum_{\mid p \leqq t} \int_{\Omega} D^{p} \varphi \overline{D^{p} \psi} d x ;
$$

die entsprechende Norm ist

$$
\|\varphi\|_{t}=+\sqrt{(\varphi, \varphi)_{t}} .
$$

Dabei ist $p=\left(p_{1}, \ldots, p_{n}\right)$ ein Multiindex mit nichtnegativen ganzen Zahlen $p_{1}, \ldots, p_{n},|p|=p_{1}+\ldots+p_{n}, \quad D^{p}=D_{1}^{p_{1}} \ldots D_{n}^{p_{n}}\left(D_{i}=\partial / \partial x_{i}\right)$.

Es sei $C_{*}^{t}(\Omega)$ die lineare Menge aller Funktionen $\varphi$ aus $C^{t}(\Omega)$ mit $\|\varphi\|_{l}<\infty$. Durch Einführung des Skalarproduktes (1.1) wird $C_{*}^{t}(\Omega) \quad$ zu einem Skalarproduktraum, dessen Vervollständigung bezüglich der Norm (1.2) mit $H^{t}(\Omega)$ bezeichnet wird (der Raum $H^{t}(\Omega)$ ist also ein Hilbertraum). Die Funktionen aus $H^{t}(\Omega)$ besitzen definitionsgemäss starke $L^{2}$-Ableitungen bis und mit Ordnung $t$ (vgl. [2], S. 3-4).

Sei $H_{0}^{t}(\Omega)$ die Abschliessung der Menge $C_{0}^{\infty}(\Omega)$ im Raume $H^{t}(\Omega)$. Es gilt $H^{0}(\Omega)=H_{0}^{0}(\Omega)=L^{2}(\Omega)$. Für $t>0$ ist $H_{0}^{t}(\Omega)$ im allgemeinen ein echter Unterraum von $H^{t}(\Omega)$ (vgl. [12], S. 15, Satz 3.1). Jede Funktion aus $C^{t}(\bar{\Omega}) \cap H_{0}^{t}(\Omega)$, zusammen mit allen ihren partiellen Ableitungen bis und mit Ordnung $t-1$, verschwindet im klassischen Sinne am Rande von $\Omega$, falls dieser genügend regulär ist. Aus diesem Grunde sagt man, dass die Funktionen aus $H_{0}^{t}(\Omega)$ und alle ihre starken $L^{2}$-Ableitungen bis und mit Ordnung $t-1$ im $L^{2}$-Sinn homogene Randwerte annehmen.

1) $C^{t}(\Omega)$ ist die Menge der $t$-mal stetig differenzierbaren komplexwertigen, auf $\Omega$ definierten Funktionen und

$$
C^{\infty}(\Omega)=\bigcap_{t=0}^{\infty} C^{t}(\Omega) .
$$

Die Menge der Funktionen aus $C^{\infty}(\Omega)$, die je einen kompakten Träger in $\Omega$ haben, bezeichnen wir mit $C_{0}^{\infty}(\Omega)$. 
Wir betrachten einen linearen partiellen Differentialausdruck

$$
l=\sum_{|p| \leqq r} a_{p} D^{p}
$$

der Ordnung $r$ mit Koeffizienten $\left.a_{p} \in L_{\mathrm{loc}}^{2}(\Omega)^{2}\right)$. Der zu $l$ formal adjungierte Ausdruck $l^{\prime}$ ist durch

$$
l^{\prime} \varphi=\sum_{|p| \leqq r}(-1)^{p \mid} D^{p}\left(\bar{a}_{p} \varphi\right)=\sum_{\mid p_{i} \leqq r} a_{p}^{*} D^{p} \varphi
$$

für alle $\varphi \in C_{0}^{\infty}(\Omega)$ als ein Differentialoperator, dessen Koeffizienten Distributionen sind, definiert. Für das Folgende setzen wir $a_{p}^{*} \in L_{\mathrm{loc}}^{2}(\Omega)$ voraus. Es gilt

$$
(l \varphi, \psi)_{0}=\left(\varphi, l^{\prime} \psi\right)_{0}
$$

für $\varphi, \psi \in C_{0}^{\infty}(\Omega)$.

Sei $f \in L^{2}(\Omega)$ gegeben. Eine Funktion $u \in L_{\text {loc }}^{2}(\Omega)$ heisst schwache Lösung der Gleichung $l u=f$, wenn die Beziehung

$$
\left(l^{\prime} \varphi, u\right)_{0}=(\varphi, f)_{0}
$$

für alle $\varphi \in C_{0}^{\infty}(\Omega)$ erfüllt ist.

Wenn wir im folgenden das verallgemeinerte Dirichletproblem mit Randdaten der Ordnung $t-1$ betrachten, wird eine Funktion $g \in L^{2}(\Omega)$ zulässig genannt, falls das für $\varphi \in C_{0}^{\infty}(\Omega)$ durch

$$
b_{g}(\varphi)=\left(l^{\prime} \varphi, g\right)_{0}
$$

definierte lineare Funktional $b_{g}$ bezüglich der Norm des Raumes $H_{0}^{t}(\Omega)$ beschränkt ist, d. h. falls $\left|\left(l^{\prime} \varphi, g\right)_{0}\right|$ für $\|\varphi\|_{t} \leqq 1$ beschränkt ist.

1.2. Wir stellen das verallgemeinerte Dirichletproblem für die Gleichung $l u=f$ mit Randdaten der Ordnung $t-1$ :

Problem I. Gegeben seien eine Funktion $f \in L^{2}(\Omega)$ und eine zulässige Funktion $g \in L^{2}(\Omega)$.

Gesucht ist eine Funktion $u$ derart, dass

$$
\left(l^{\prime} \varphi, u\right)_{0}=(\varphi, f)_{0}
$$

für alle $\varphi \in C_{0}^{\infty}(\Omega)$ gilt (d.h. u eine schwache Lösung der Gleichung $l u=f$ ) und dass

$$
v=u-g \in H_{0}^{t}(\Omega)
$$

ist.

$\left.{ }^{2}\right)$ Eine Funktion $u$ liegt definitionsgemäss in $L_{\text {loc }}^{2}(\Omega)$, falls zu jedem Punkt $x \in \Omega$ eine (offene) Umgebung $U$ mit $\left.\quad u\right|_{U} \in L^{2}(U)$ existiert. 
Mit $\widetilde{b_{g}}$ bezeichnen wir die stetige Erweiterung des durch (1.5) definierten Funktionals $b_{g}$ auf den Raum $H_{0}^{t}(\Omega)$ : Es gilt also

$$
\widetilde{b_{g}}(\varphi)=b_{g}(\varphi)=\left(l^{\prime} \varphi, g\right)_{0}
$$

für $\varphi \in C_{0}^{\infty}(\Omega)$.

1.3. Problem I ist äquivalent dem folgenden

Problem II. Gegeben seien eine Funktion $f \in L^{2}(\Omega)$ und eine zulässige Funktion $g \in L^{2}(\Omega)$.

Gesucht ist eine solche Funktion $v \in H_{0}^{t}(\Omega)$, dass

$$
\left(l^{\prime} \varphi, v\right)_{0}=(\varphi, f)_{0}-\left(l^{\prime} \varphi, g\right)_{0}
$$

für alle $\varphi \in C_{0}^{\infty}(\Omega)$ gilt.

Wegen der für $g$ gemachten Voraussetzung ist das für Funktionen $\varphi \in C_{0}^{\infty}(\Omega)$ definierte lineare Funktional

$$
a(\varphi)=(\varphi, f)_{0}-\left(l^{\prime} \varphi, g\right)_{0}
$$

bezüglich der Topologie von $H_{0}^{t}(\Omega)$ beschränkt. Nach dem Darstellungssatz von M. Fréchet und F. Riesz existiert ein durch $f$ und $g$ eindeutig bestimmtes Element $w \in H_{0}^{\prime}(\Omega)$ derart, dass für alle $\varphi \in C_{0}^{\infty}(\Omega)$

$$
a(\varphi)=(\varphi, f)_{0}-\left(l^{\prime} \varphi, g\right)_{0}=(\varphi, w)_{t}
$$

gilt.

Sei $\varphi$ eine feste Funktion aus $C_{0}^{\infty}(\Omega)$. Für alle $v \in H_{0}^{t}(\Omega)$ gilt dann die Ungleichung

$$
\left|\left(l^{\prime} \varphi, v\right)_{0}\right| \leqq c(\varphi)\|v\|_{0} \leqq c(\varphi)\|v\|_{t}
$$

mit einer positiven, von $v$ unabhängigen Konstante $c(\varphi)$. Also ist das antilineare Funktional

$$
d_{\varphi}(v)=\left(l^{\prime} \varphi, v\right)_{0}
$$

im Raum $H_{0}^{t}(\Omega)$ beschränkt, und nach dem oben erwähnten Satz gibt es ein solches Element $z_{\varphi} \in H_{0}^{t}(\Omega)$, dass $\left(l^{\prime} \varphi, v\right)_{0}=\left(z_{\varphi}, v\right)_{t}$ für alle $v \in H_{0}^{t}(\Omega)$ gilt. Durch $T \varphi=z_{\varphi}$ definieren wir einen linearen Operator $T$ in $H_{0}^{t}(\Omega)$ mit Definitionsbereich $C_{0}^{\infty}(\Omega)$. Es gilt also

$$
\left(l^{\prime} \varphi, v\right)_{0}=(T \varphi, v)_{t}
$$

für alle $v \in H_{0}^{t}(\Omega)$. Berücksichtigen wir (1.9) und (1.10), so erhalten wir eine den Problemen I und II gleichwertige Fragestellung:

Problem III. Sei $w$ das durch (1.9) festgelegte Element aus $H_{0}^{t}(\Omega)$, und sei $T$ der durch (1.10) definierte lineare Operator des Raumes $H_{0}^{t}(\Omega)$. 
Gesucht ist eine Funktion $v \in H_{0}^{t}(\Omega)$ derart, dass

$$
(T \varphi, v)_{t}=(\varphi, w)_{t}
$$

für alle $\varphi \in C_{0}^{\infty}(\Omega)$ gilt.

Durch die Beziehung (1.10) haben wir dem Differentialausdruck $l$ den Operator $T$ zugeordnet. Dadurch ist unsere Fragestellung in den Raum $H_{0}^{t}(\Omega)$ der Funktionen mit »homogenen» Randdaten verlagert worden. Im folgenden werden unsere Betrachtungen in diesem Hilbertraum durchgeführt.

Aus (1.11) folgt

Satz 1.1. Problem III ist lösbar dann und nur dann, wenn die Funktion $w$ in der Wertemenge des im Hilbertraum $H_{0}^{t}(\Omega)$ zu $T$ adjungierten Operators $T^{*}$ liegt.

\section{Hilfssätze über die Wertemenge abgeschlossener Operatoren eines Hilbertraumes}

2.1. Es sei $S$ ein (nicht notwendigerweise beschränkter) linearer selbstadjungierter Operator des Hilbertraumes $\mathfrak{\mathfrak { H }}$. Mit $K(S)$ bezeichnen wir den Kern, mit $D(S)$ die Definitionsmenge und mit $W(S)$ die Wertemenge von $S$.

Der Operator $S$ induziert bekanntlich die orthogonale Zerlegung:

$$
\mathfrak{\mathfrak { H }}={\mathfrak{\mathfrak { H } _ { 0 }}}_{\mathbf{C}} \oplus \tilde{\mathfrak{H}}_{1}
$$

von $\mathfrak{S}$, wobei

ist.

$$
\check{\mathfrak{S}}_{0}=K(S), \quad \check{\mathfrak{Y}}_{1}=\overline{W(S)}
$$

Mit Hilfe der Spektralzerlegung

$$
S=\int_{-\infty}^{+\infty} \lambda d E_{\lambda}
$$

definieren wir die lineare Menge

$$
\mathfrak{M}=\left\{u \in \mathfrak{S} \mid \int_{-\infty}^{+\infty} \lambda^{-2} d\left(E_{\lambda} u, u\right)<\infty\right\} .
$$

Die Menge $\mathfrak{M}$ ist in $\check{\mathfrak{S}}_{1}$ enthalten.

Es gilt

Satz 2.1. Die Wertemenge $W(S)$ des selbstadjungierten Operators $S$ fällt mit der linearen Menge $\mathfrak{M}$ zusammen. 
Dieser Satz wurde für beschränkte selbstadjungierte Operatoren von F. E. Browder [4] bewiesen. Für unbeschränkte selbstadjungierte Operatoren $S$ wird die Inklusion $W(S) \subset \mathfrak{M}$ wie im Falle eines beschränkten Operators gezeigt (vgl. auch [18], S. 6-8). Der Beweis der Gleichheit $W(S)=\mathfrak{M}$ lässt sich aber nicht direkt übertragen; er sei hier deshalb ausgeführt:

Sei $R$ der durch

$$
R=\int_{-\infty}^{+\infty} \lambda^{-1} d E_{\lambda}
$$

auf der Menge $D(R)=\mathfrak{M}$ definierte Operator. Da der Raum $\mathfrak{H}_{1} \quad$ von jedem Projektor $E_{\lambda}$ in sich abgebildet wird, ist wegen $D(R)=\mathfrak{M} \subset \mathfrak{H}_{1}$ die Menge $W(R)$ in $\mathfrak{\mathscr { S }}_{1}$ enthalten. Der Theorie der Funktionen selbstadjungierter Operatoren (vgl. z. B. [1], S. 210-219) entnehmen wir, dass $R$ ein selbstadjungierter Operator im Hilbertraum $\breve{\mathfrak{S}}_{1}$ ist.

Mit $S_{1}$ bezeichnen wir die Einschränkung von $S$ auf $\mathfrak{\mathfrak { S }}_{1}$. Der Operator $S_{1}$ ist in $\tilde{\mathfrak{D}}_{1}$ selbstadjungiert, und die Zahl 0 ist nicht Eigenwert von $S_{1}$. Es existiert also der selbstadjungierte Operator $\left(S_{1}\right)^{-1}$, der auf der linearen Menge $W\left(S_{1}\right)$ definiert ist.

Es sei $u$ bzw. $v$ ein beliebiges Element aus $\mathfrak{M}$ bzw. $D\left(S_{1}\right)$. Nach Definition (2.2) ist

$$
\left(R u, S_{1} v\right)=(R u, S v)=\int_{-\infty}^{+\infty} \lambda^{-1} d\left(E_{\lambda} u, S v\right) .
$$

Da

$$
\begin{aligned}
\left(E_{\lambda} u, S v\right)=\overline{\left(S v, E_{i} u\right)} & =\int_{-\infty}^{+\infty} \xi d_{\xi} \overline{\left(E_{\xi} v, E_{\lambda} u\right)} \\
& =\int_{-\infty}^{\lambda} \xi d_{\xi}\left(E_{\xi} u, v\right)
\end{aligned}
$$

und folglich

$$
d_{\lambda}\left(E_{\lambda} u, S v\right)=\lambda_{i} d_{i}\left(E_{i} u, v\right)
$$

ist, ergibt sich

$$
\left(R u, S_{1} v\right)=\int_{-\infty}^{+\infty} d_{\lambda_{\lambda}}\left(E_{i} u, v\right)=(u, v) .
$$

Auf Grund der Relation $W(S)=W\left(S_{1}\right) \subset \mathfrak{M} \quad$ gilt

$$
\left(u, R S_{1} v\right)=\left(R u, S_{1} v\right)=(u, v)
$$


für alle $u \in \mathfrak{M}, v \in D\left(S_{1}\right)$. Wir folgern hieraus, dass

$$
R \supset\left(S_{1}\right)^{-1}
$$

ist. Da die Operatoren $R$ und $\left(S_{1}\right)^{-1}$ selbstadjungiert im Raume $\tilde{\mathfrak{S}}_{1}$ sind, folgt deren Identität und daraus

$$
\mathfrak{M}=D(R)=D\left(\left(S_{1}\right)^{-1}\right)=W\left(S_{1}\right)=W(S),
$$

w. z. b. w.

2.2. Es sei $A$ ein in dem Hilbertraum $\mathfrak{H}$ dicht definierter abgeschlossener linearer Operator. Dann ist der Operator $A A^{*}$ selbstadjungiert und positiv. Das folgende Lemma von J. von Neumann [20] erlaubt uns die Beschreibung der Wertemenge von $A$ mit Hilfe von Satz 2.1.

Lemma 2.2. Es seien $A$ und $B$ zwei dicht definierte abgeschlossene lineare Operatoren des Raumes $\mathfrak{H}$ derart, dass

$$
A A^{*}=B B^{*}
$$

ist. Dann existiert ein auf $\mathfrak{\mathfrak { B }}$ definierter beschränkter linearer Operator $\checkmark$ mit

$$
B=A V, \quad A=B V^{*} \text {. }
$$

Aus diesem Lemma folgt unmittelbar

Korollar 2.3. Falls die Operatoren $A$ und $B$ die Voraussetzungen von Lemma 2.2 erfüllen, fallen ihre Wertemengen zusammen.

Es gilt (vgl. S. Stenholm [28])

Satz 2.4. Sei $A$ ein dicht definierter abgeschlossener linearer Operator des Raumes $\mathfrak{\mathfrak { S }}$. Ferner sei

$$
A A^{*}=\int_{-0}^{\infty} \lambda d E_{\lambda}
$$

die Spektralzerlegung des selbstadjungierten Operators $A A^{*}$. Damit ein Element $u \in \mathfrak{S}$ in der Wertemenge $W(A)$ von $A$ liegt, ist notwendig und hinreichend, dass

$$
\int_{-0}^{\infty} \lambda^{-1} d\left(E_{i} u, u\right)<\infty
$$

gilt.

Beweis. Nach Korollar 2.3 haben $A$ und die positive selbstadjungierte Quadratwurzel $B$ aus $A A^{*}$, 


$$
B=\int_{-0}^{\infty} \lambda^{\frac{1}{2}} d E_{\lambda}
$$

dieselbe Wertemenge. Gemäss Satz 2.1 liegt ein Element $u$ genau dann in $W(B)$, wenn die Bedingung (2.4) erfüllt ist.

Der folgende Satz verallgemeinert Resultate von R. Nevanlinna [25] und I. S. Louhivaara [16]:

Satz 2.5. $\quad E s$ sei $A$ ein dicht definierter abgeschlossener linearer Operator des Raumes $\mathfrak{H}$, und es bezeichne $\left\{E_{\lambda}\right\}$ die durch den selbstadjungierten Operator $A A^{*}$ erzeugte Spektralschar. Für jede nichtnegative Zahl $\varkappa$ sei der lineare Unterraum $\mathfrak{S}^{*}$ von $\mathfrak{\mathfrak { H }}$ durch

$$
\mathfrak{\mathfrak { S }}^{\varkappa}=E_{\varkappa} \mathfrak{H}_{\mathfrak{S}}
$$

definiert.

a) Notwendig dafür, dass ein Element $u \in \mathfrak{\mathfrak { g }}$ in $W(A)$ liegt, ist die Bedingung

$$
(z, u)=0 \quad \text { für alle } z \in \mathfrak{S}^{+0} .
$$

b) Hinreichend dafür, dass ein Element $u \in \mathfrak{H}$ in $W(A)$ liegt, ist die Existenz einer positiven Zahl $\varkappa$ derart, dass

$$
(z, u)=0 \quad \text { für alle } z \in \mathfrak{S}^{*}
$$

gilt.

Beweis. a) Die Notwendigkeit von (2.5) folgt aus der orthogonalen Zerlegung

$$
\tilde{\mathfrak{H}}=K\left(A^{*}\right) \oplus \overline{\mathrm{W}(A)}
$$

von $\mathfrak{\mathfrak { g }}$ unter Berücksichtigung der Relation

$$
K\left(A^{*}\right)=K\left(A A^{*}\right)=\check{\mathfrak{g}}^{-0} .
$$

b) Falls $u$ orthogonal auf $\mathfrak{S}^{*}(\varkappa>0)$ ist, verschwindet $\left(E_{j} u, u\right)$ identisch im Intervall $-0 \leqq \lambda \leqq \varkappa$; es gilt deshalb

$$
\int_{-0}^{\infty} \lambda^{-1} d\left(E_{\lambda} u, u\right)=\int_{\lambda}^{\infty} \lambda^{-1} d\left(E_{\lambda} u, u\right)<\infty,
$$

und nach Satz 2.4 liegt $u$ in der Menge $W(A)$. 


\section{Kriterien für die Lösbarkeit von Problem III}

3.1. Es sei $T$ der durch (1.10) definierte lineare Operator des Hilbertraumes $H_{0}^{t}(\Omega)$.

Wir zeigen zuerst, dass der Operator $T^{* *}$ definiert ist: $\mathrm{Zu}$ jeder Funktion $\psi \in C_{0}^{\infty}(\Omega)$ existiert eine Zahl $C_{\psi}(\geqq 0)$ derart, dass

$$
\left|(T \varphi, \psi)_{t}\right|=\left|\left(l^{\prime} \varphi, \psi\right)_{0}\right|=\left|(\varphi, l \psi)_{0}\right| \leqq c_{\psi}\|\varphi\|_{0} \leqq c_{\psi}\|\varphi\|_{t}
$$

für alle $\varphi \in C_{0}^{\infty}(\Omega)$ gilt; das durch

$$
\gamma_{\psi}(\varphi)=(T \varphi, \psi)_{t}
$$

für alle $\varphi \in C_{0}^{\infty}(\Omega)$ erklärte Funktional ist also im Raum $H_{0}^{t}(\Omega)$ beschränkt. Folglich liegt $\psi$ in $D\left(T^{*}\right)$, und $T^{*}$ ist dicht in $H_{0}^{t}(\Omega)$ definiert. Dadurch existiert der Operator $T^{* *}$, und dieser fällt mit der Abschliessung von $T$ zusammen.

3.2. Wendet man den Satz 2.4 auf den Operator $A=T^{*}$ an und berücksichtigt man den Satz 1.1, so erhält man die folgende Verallgemeinerung eines Satzes von F. E. Browder [4]:

Satz 3.1. $\quad$ Es sei $T$ der durch (1.10) definierte Operator des Hilbertraumes $H_{0}^{t}(\Omega)$. Der selbstadjungierte Operator $T^{*} T^{* *}$ besitze die Spektralzerlegung

$$
T^{*} T^{* *}=\int_{-0}^{\infty} \lambda d E_{\lambda} .
$$

Problem III ist dann und nur dann lösbar, wenn die durch (1.9) erklärte Funktion $w$ der Bedingung

$$
\int_{-0}^{\infty} \lambda^{-1} d\left(E_{\lambda} w, w\right)<\infty
$$

genügt.

Aus den Sätzen 1.1 und 2.5 ergibt sich als eine Erweiterung eines Kriteriums von I. S. Louhivaara [17] folgender

Satz 3.2. Der Operator $T$ sei durch (1.10) definiert, und $T^{*} T^{* *}$ besitze die Spektralzerlegung (3.1). Für jede Zahl $\varkappa(\geqq 0)$ sei der Unterraum $\mathfrak{H}^{*}$ von $H_{0}^{t}(\Omega)$ durch

$$
\mathfrak{S}^{*}=E_{\varkappa} H_{0}^{t}(\Omega)
$$

erklärt. 
a) Notwendig für die Lösbarkeit von Problem III ist die Gültigkeit der Beziehung

$$
(z, w)_{t}=(z, f)_{0}-\widetilde{b_{g}}(z)=0 \quad \text { für alle } z \in \mathfrak{S}^{+0} .
$$

b) Hinreichend für die Lösbarkeit von Problem III ist die Existenz einer positiven Zahl $x$ derart, dass

$$
(z, w)_{t}=(z, f)_{0}-\widetilde{b_{g}}(z)=0 \quad \text { für alle } \quad z \in \mathfrak{S}^{*}
$$

gilt.

3.3. Wir wollen nun eine spezielle Klasse von Differentialausdrücken betrachten, für die die Bedingungen a) und b) in Satz 3.2 zusammenfallen.

Satz 3.3. Es sei $l$ ein im Gebiet $\Omega\left(\subset R^{n}\right)$ definierter linearer Differentialausdruck der Form (1.3). Wir setzen voraus:

(*) $\quad Z u \quad l$ existiert eine positive Zahl $\delta$ derart, dass jede lineare Menge $G \subset C_{0}^{\infty}(\Omega)$, die der Bedingung

$$
\left|\operatorname{Re}(\varphi, l \varphi)_{0}\right| \leqq \delta\|\varphi\|_{t}^{2} \quad \text { für alle } \quad \varphi \in G
$$

genügt, endliche Dimension hat.

Dann ist die Gültigkeit der Beziehung

$$
(z, w)_{t}=(z, f)_{0}-\widetilde{b}_{g}(z)=0 \quad \text { für alle } z \in \mathfrak{S ূ}^{+0}
$$

notwendig und hinreichend für die Lösbarkeit von Problem III.

Beweis. Der Differentialausdruck $l$ erfülle die Voraussetzung (*). Wir beweisen indirekt, dass im Intervall $\left(-\delta^{2} / 4,+\delta^{2} / 4\right)$ keine Punkte des wesentlichen Spektrums von $T^{*} T^{* *}$ liegen. Unter der Annahme, dass es in diesem Intervall solche Punkte gibt, ist die Ungleichung

$$
\left\|T^{*} T^{* *} u\right\|_{t} \leqq \frac{\delta^{2}}{4}\|u\|_{t}
$$

für eine von unendlichvielen paarweise orthogonalen Vektoren $u_{i} \in D\left(T^{*} T^{* *}\right) \quad\left(i=1,2, \ldots,\left\|u_{i}\right\|_{t}=1\right)$ und allen endlichen Linearkombinationen dieser Vektoren gebildete lineare Menge $M$ erfüllt ([21], S. 309). Aus (3.6) ergibt sich

$$
\left|\left(T^{*} T^{* *} u, u\right)_{t}\right| \leqq \frac{\delta^{2}}{4}\|u\|_{t}^{2}
$$

und folglich

$$
\left\|T^{* *} u\right\|_{t} \leqq \frac{\delta}{2}\|u\|_{t}
$$


für alle $u \in M$. Zu jedem Basisvektor $u_{i}$ von $M\left(u_{i} \in D\left(T^{* *}\right)\right)$ existiert eine Folge $\left\{\varphi_{i}^{j}\right\}_{j=1}^{\infty} \subset C_{0}^{\infty}(\Omega)$ derart, dass $\varphi_{i}^{j} \rightarrow u_{i}$ und $T \varphi_{i}^{j} \rightarrow T^{* *} u_{i}$ in $H_{0}^{t}(\Omega)$. Sei $\gamma=\delta /(2+2 \delta)$. Wir wählen aus jeder Folge $\left\{\phi_{i}^{j}\right\}$ ein Element $\varphi_{i}$ so, dass sowohl $\left\|\varphi_{i}-u_{i}\right\|_{t} \leqq \gamma / 2^{i}$ als auch $\left\|T \varphi_{i}-T^{* *} u_{i}\right\|_{t}$ $\leqq \gamma / 2^{i} \quad(i=1,2, \ldots)$ ist. Jedem Vektor

ordnen wir den Vektor

$$
u=\sum_{i=1}^{k} \lambda_{i} u_{i}
$$

$$
\varphi=\sum_{i=1}^{k} \lambda_{i} \varphi_{i}
$$

zu. Die Elemente $\varphi$ spannen eine lineare Menge $G\left(\subset C_{0}^{\infty}(\Omega)\right)$ auf. Diese ist unendlichdimensional, da die Elemente $\varphi_{i}$ eine nicht relativ kompakte beschränkte Menge bilden. Bei dieser Zuordnung gelten die Relationen:

$$
\begin{gathered}
(1-\gamma)\|u\|_{t} \leqq\|\varphi\|_{t} \leqq(1+\gamma)\|u\|_{t} \\
\left\|T^{* *} u\right\|_{t}-\gamma\|u\|_{t} \leqq\|T \varphi\|_{t} \leqq\left\|T^{* *} u\right\|_{t}+\gamma\|u\|_{t} .
\end{gathered}
$$

Wir beweisen die Gültigkeit von (3.8), analog kann (3.9) gezeigt werden. Auf Grund der Orthonormiertheit der Funktionen $u_{i}$ gilt die Besselsche Gleichung

also ist

$$
\sum_{i=1}^{k}\left|\lambda_{i}\right|^{2}=\|u\|_{t}^{2}
$$

$$
\left|\lambda_{i}\right| \leqq\|u\|_{t}
$$

und es ergibt sich die Abschätzung (3.8):

$$
\|\varphi-u\|_{t} \leqq \sum_{i=1}^{k}\left|\lambda_{i}\right|\left\|\varphi_{i}-u_{i}\right\|_{t} \leqq\left(\sum_{i=1}^{k} \frac{1}{2^{i}}\right) \gamma\|u\|_{t} \leqq \gamma\|u\|_{t} .
$$

Aus (3.8) folgt

$$
\frac{1}{1+\gamma}\|\varphi\|_{t} \leqq\|u\|_{t} \leqq \frac{1}{1-\gamma}\|\varphi\|_{t}
$$

und damit unter Berücksichtigung von (3.9) und (3.7) sowie der Wahl von $\gamma$

$$
\begin{aligned}
\|T \varphi\|_{t} & \leqq\left\|T^{* *} u\right\|_{t}+\gamma\|u\|_{t} \leqq\left(\frac{\delta}{2}+\gamma\right)\|u\|_{t} \\
& \leqq\left(\frac{\delta}{2}+\gamma\right) \frac{1}{1-\gamma}\|\varphi\|_{t}=\delta\|\varphi\|_{t}
\end{aligned}
$$


es gilt somit

$$
\left|\operatorname{Re}(\varphi, l \varphi)_{0}\right|=\left|\operatorname{Re}(T \varphi, \varphi)_{t}\right| \leqq \delta\|\varphi\|_{t}^{2}
$$

für alle $\varphi \in G$. Entgegen der Voraussetzung haben wir die Existenz einer unendlichdimensionalen linearen Menge $G \subset C_{0}^{\infty}(\Omega)$ gezeigt, die (3.5) erfüllt. Also liegen keine Punkte des wesentlichen Spektrums von $T^{*} T^{* *}$ im Intervall $\left(-\delta^{2} / 4,+\delta^{2} / 4\right)$.

Da der Operator $T^{*} T^{* *}$ im betrachteten Intervall nur endlich viele Eigenwerte (endlicher Vielfachheit) besitzt, gibt es eine positive Zahl $\%$ derart, dass $\mathfrak{\mathfrak { S }}^{+0}=\mathfrak{S}^{*}$ ist, womit Satz 3.3 bewiesen ist.

3.4. Als Vorbereitung zur Anwendung der Theorie auf elliptische Differentialausdrücke geben wir eine leichter verifizierbare Bedingung an, die die Gültigkeit der Voraussetzung (*) von Satz 3.3 garantiert.

Lemma 3.4. Sei $l$ ein in einem beschränkten Gebiet $\Omega\left(\subset R^{n}\right)$ erklärter linearer Differentialausdruck der Form (1.3), und sei $t>0$. Falls zwei reelle Konstanten $c_{0}>0$ und $k_{0}$ derart existieren, dass

$$
\operatorname{Re}(\varphi, l \varphi)_{0} \geqq c_{0}\|\varphi\|_{t}^{2}-k_{0}\|\varphi\|_{0}^{2}
$$

für alle $\phi \in C_{0}^{\infty}(\Omega)$ gilt, genügt der Differentialausdruck $l$ der Voraussetzung (*) von Satz 3.3 mit $\delta=c_{0} / 2$.

Beweis. Es sei $G\left(\subset C_{0}^{\infty}(\Omega)\right)$ eine lineare Menge, die die Ungleichung (3.5) mit $\delta=c_{0} / 2$ erfüllt. Dann gilt

$$
\operatorname{Re}(\varphi, l \varphi)_{0}+k_{0}\|\varphi\|_{0}^{2}-\frac{c_{0}}{2}\|\varphi\|_{t}^{2} \leqq k_{0}\|\varphi\|_{0}^{2}
$$

für alle $\varphi \in G$, und aus (3.11) ergibt sich

$$
\operatorname{Re}(\varphi, l \varphi)_{0}+k_{0}\|\varphi\|_{0}^{2}-\frac{c_{0}}{2}\|\varphi\|_{t}^{2} \geqq \frac{c_{0}}{2}\|\varphi\|_{t}^{2}
$$

für alle $\varphi \in C_{0}^{\infty}(\Omega)$. Gemäss (3.12) und (3.13) gilt auf $G$ die Ungleichung

$$
\frac{c_{0}}{2}\|\varphi\|_{t}^{2} \leqq k_{0}\|\varphi\|_{0}^{2}
$$

Es sei $G_{1}$ die Menge der Funktionen $\varphi$ aus $G$ mit $\|\varphi\|_{0}=1$. Wegen (3.14) ist $G_{1}$ die Einbettung einer im Raum $H_{0}^{t}(\Omega)$ beschränkten Menge in den Raum $L^{2}(\Omega)$. Da diese Einbettung kompakt ist (vgl. [2], Satz 8.3), ist die "Einheitssphäre» $G_{1}$ in $L^{2}(\Omega)$ relativ kompakt. Also muss $G$ endlichdimensional sein. 


\section{Anwendung auf gleichmässig stark elliptische Differentialausdrücke}

4.1. Für die Anwendungen in diesem Kapitel benötigen wir einige Definitionen, die wir nun zusammenstellen werden.

Es sei $l$ ein linearer Differentialausdruck der Form (1.3),

$$
l=\sum_{\mid p \leqq \leqq r} a_{p} D^{p} \quad\left(a_{p} \in L_{\mathrm{loc}}^{2}(\Omega)\right),
$$

und es bezeichne $l^{\prime}$ den zu $l$ formal adjungierten Ausdruck.

Die durch die Ausdrücke $l$ und $l^{\prime}$ auf der Menge $C_{0}^{\infty}(\Omega)$ definierten Operatoren seien $l_{0}$ bzw. $l_{0}^{\prime}$. Da in $L^{2}(\Omega)$ die Beziehung ${ }^{3}$ )

$$
l_{0} \subset\left(l_{0}^{\prime}\right)^{+}
$$

gilt, ist der Operator $l_{0}$ abschliessbar.

Die durch $l$ erzeugten Operatoren $L_{\min }$ und $L_{\max }$ des Raumes $L^{2}(\Omega)$ sind durch

$$
L_{\min }=l_{0}^{++} \quad \text { bzw. } \quad L_{\max }=\left(l_{0}^{\prime}\right)^{+}
$$

definiert. Entsprechend sind die Operatoren $L_{\min }^{\prime}$ und $L_{\max }^{\prime}$ erklärt:

$$
L_{\min }^{\prime}=\left(l_{0}^{\prime}\right)^{++}, \quad L_{\max }^{\prime}=l_{0}^{+} .
$$

Aus (4.1) folgt

$$
L_{\min } \subset L_{\max } .
$$

Der Operator $L$ bzw. $L^{\prime}$ ist die Einschränkung von $L_{\max }$ bzw. $L_{\max }^{\prime} \quad$ auf die Menge $D\left(L_{\max }\right) \cap H_{0}^{t}(\Omega)$ bzw. $D\left(L_{\max }^{\prime}\right) \cap H_{0}^{t}(\Omega)$. Gerade mit diesen zuletzt definierten Operatoren $L$ und $L^{\prime}$ werden wir in den nachstehenden Abschnitten arbeiten.

4.2. Wir beweisen zuerst das folgende

Lemma 4.1. $\quad$ Es sei $A$ ein dicht definierter linearer Operator eines Hilbertraumes $\mathfrak{\mathfrak { B }}$. Der Operator $A$ lasse die Abschliessung $\bar{A} z u$, und auf $W(A)$ existiere das beschränkte Inverse $A^{-1}$. Dann gilt $\mathrm{I}^{*}\left(A^{*}\right)=\mathfrak{H}$.

Beweis. Die Wertemenge $W(\bar{A})$ ist unter den Voraussetzungen ein (abgeschlossener) Unterraum von $\mathfrak{h}$ (vgl. [27], S. 475, Satz 2). Wir zeigen, dass zu einem beliebigen Element $v^{*} \in \mathfrak{H}$ ein Element $v \in \mathfrak{H}$ derart existiert, dass

$\left.{ }^{3}\right)$ Mit $A^{+}$wird im folgenden der zu $A$ im Raume $L^{2}(\Omega)$ adjungierte Operator bezeichnet. 


$$
\left(u, v^{*}\right)=(\bar{A} u, v)
$$

für alle $u \in D(\bar{A})$ gilt. Mit $z=\bar{A} u$ erhalten wir

$$
\left(u, v^{*}\right)=\left(\bar{A}^{-1} z, v^{*}\right) .
$$

Da der Operator $\bar{A}^{-1}$ auf $W(\bar{A})$ beschränkt ist, ist das durch

$$
f_{v^{*}}(z)=\left(\bar{A}^{-1} z, v^{*}\right)
$$

auf $W(\bar{A})$ erklärte lineare Funktional $f_{v^{*}}$ beschränkt. Nach dem Darstellungssatz von M. Fréchet und F. Riesz existiert ein Element $v \in W(\bar{A})$ so, dass

$$
f_{v^{*}}(z)=(z, v)
$$

für alle $z \in W(\bar{A})$ gilt. Es ergibt sich also

$$
\left(u, v^{*}\right)=f_{v^{*}}(z)=(z, v)=(\bar{A} u, v)
$$

für alle $u \in D(\bar{A})$, w. z. b. w.

4.3. Bevor wir durch Spezialisierung unserer allgemeinen Sätze aus Kapitel 3 Lösbarkeitsbedingungen für gleichmässig stark elliptische Differentialgleichungen herleiten, erwähnen wir ohne Beweis bekannte Resultate $\left.^{4}\right)$.

Es sei

$$
l=\sum_{|p|, \mid q \leqq m}(-1)^{|p|} D^{p} a_{p q} D^{q}
$$

ein linearer partieller Differentialausdruck der Ordnung $2 \mathrm{~m}$, der den folgenden Voraussetzungen genügt ${ }^{5}$ ):

1) Die komplexwertigen Koeffizienten $a_{p q}$ sind beschränkt und messbar in $\Omega$.

2) Die Funktionen $a_{p q}$ sind für $|p|=|q|=m$ gleichmässig stetig in $\Omega$.

3) Der Ausdruck $l$ ist gleichmässig stark elliptisch in $\Omega$, d. h. es existiert eine positive Zahl $C$ derart, dass

$$
\operatorname{Pe}(-1)^{m} \sum_{|\boldsymbol{p}|=|\boldsymbol{q}|=m} a_{p q}(x) \xi^{p} \xi^{q} \geqq C \mid \xi^{2 m}
$$

für alle reellen Vektoren $\xi=\left(\xi_{1}, \ldots, \xi_{n}\right)$ und alle $x \in \Omega$ gilt. Dabei ist $\mid \xi^{2}=\xi_{1}^{2}+\ldots+\xi_{n}^{2}$ und $\xi^{p}=\xi_{1}^{p_{1}} \ldots \xi_{n}^{p_{n}}, \quad p=\left(p_{1}, \ldots, p_{n}\right)$.

4) Wegen der Beweise sei z. B. auf [2], [3], [6] verwiesen.

5) Die formale Selbstadjungiertheit des Differentialausdruckes $\left(a_{p q}=\overline{a_{q p}}\right)$ wird nicht vorausgesetzt. 
Auf Grund der Voraussetzung 1) gibt es eine solche Konstante $K$, dass

$$
\left|\sum_{|p| q, \mid \leq m}\left(D^{p} \varphi, a_{p q} D^{q} \psi\right)_{0}\right| \leqq K\|\varphi\|_{m}\|\psi\|_{m}
$$

für alle $\varphi, \psi \in C_{*}^{m}(\Omega)$ ist; die für Funktionen $\varphi, \psi \in C_{*}^{m}(\Omega)$ definierte Bilinearform

$$
B(\varphi, \psi)=\sum_{|p|, q \mid \leq m}\left(D^{p} \varphi, a_{p q} D^{q} \psi\right)_{0}
$$

ist also beschränkt im Raum $H^{m}(\Omega)$. Mit $\tilde{B}(u, v)$ bezeichnen wir die stetige Erweiterung dieser Form auf den Raum $H^{m}(\Omega)$; es gilt dann offenbar die Ungleichung

$$
|\tilde{B}(u, v)| \leqq K\|u\|_{m}\|v\|_{m}
$$

für alle $u, v \in H^{m}(\Omega)$. Die Form $B$ (bzw. $\tilde{B}$ ) ist eine der zum Ausdruck $l$ gehörenden Dirichletschen Bilinearformen.

Die Voraussetzungen 1)-3) implizieren die Gültigkeit einer »Gårdingschen Ungleichung»: Es gibt zwei reelle Konstanten $c_{0}>0$ und $k_{0}$ so, dass

$$
\operatorname{Re}(\varphi, l \varphi)_{0}=\operatorname{Re}\left(\varphi, l^{\prime} \varphi\right)_{0}=\operatorname{Re} B(\varphi, \varphi) \geqq c_{0}\|\varphi\|_{m}^{2}-k_{0}\|\varphi\|_{0}^{2}
$$

für alle $\varphi \in C_{0}^{\infty}(\Omega)$ ist. Durch stetige Erweiterung im Raume $H_{0}^{m}(\Omega)$ ergibt sich

$$
\operatorname{Re} \tilde{B}(u, u) \geqq c_{0}\|u\|_{m}^{2}-k_{0}\|u\|_{0}^{2}
$$

für alle $u \in H_{0}^{m}(\Omega)$.

Mit Hilfe des Darstellungssatzes von P. D. Lax und A. N. Milgram [11] sowie der Riesz - Schauderschen Theorie sind die folgenden Resultate über die Lösbarkeit von Problem I mit $t=m$ hergeleitet worden $\left.{ }^{6}\right)$ :

Satz 4.2. Es sei $l$ ein Differentialausdruck der Form (4.4), der die Voraussetzungen 1)-3) erfüllt. Es bezeichne $\tilde{B}(u, v)$ die stetige Erweiterung der für Funktionen aus $C_{*}^{m}(\Omega)$ durch (4.5) definierten Bilinearform $B$ auf den Raum $H^{m}(\Omega)$.

a) Falls $\operatorname{Re} \lambda \geqq k_{0}$ ist (wobei $k_{0}$ die in der Ungleichung (4.\%) vorkommende Konstante bezeichnet), hat Problem I für die Differentialgleichung

$$
(l+\lambda) u=f
$$

bei beliebiger Wahl der Funktionen $f \in L^{2}(\Omega)$ und $g \in H^{m}(\Omega)$ eine und nur eine Lösung.

$\left.{ }^{6}\right)$ Das durch (1.5) für $\varphi \in C_{0}^{\infty}(\Omega)$ erklärte Funktional $b_{g}$ ist für $g \in H^{m}(\Omega)$ wegen $b_{g}(\varphi)=\left(l^{\prime} \varphi, g\right)_{0}=\tilde{B}(\varphi, g)$ bezüglich der Norm des Raumes $H_{0}^{m}(\Omega)$ beschränkt; d. h. jedes Element $g \in H^{m}(\Omega)$ ist zulässig. 
b) Ist das Gebiet $\Omega$ beschränkt, so gilt der Fredholmsche Alternativsatz: Die Gleichungen $l u=0$ und $l^{\prime} v=0$ haben dieselbe endliche Anzahl $h$ linear unabhängiger schwacher Lösungen mit "homogenen Randdaten"

$$
u_{1}, \ldots, u_{h} \in H_{0}^{m}(\Omega) \quad \text { bzw. } \quad v_{1}, \ldots, v_{h} \in H_{0}^{m}(\Omega) .
$$

Problem I für die Gleichung $l u=f$ hat eine (bis auf eine Linearkombination der Funktionen $u_{1}, \ldots, u_{h}$ eindeutig bestimmte) schwache Lösung dann und nur dann, wenn

$$
\left(v_{j}, f\right)_{0}-\tilde{B}\left(v_{j}, g\right)=0
$$

für $j=1, \ldots, h$ gilt.

4.4. Wir wollen nun, von den für allgemeinere Differentialausdrücke gültigen Resultaten der Kapitel 1 und 3 ausgehend, eine Erweiterung von Satz 4.2 beweisen: auf die Voraussetzung der Beschränktheit der Koeffizienten wird im folgenden verzichtet.

Satz 4.3. Der Ausdruck

$$
l=\sum_{|p| \leq 2 m} a_{p} D^{p}
$$

erfülle die folgenden Voraussetzungen:

i) Die Koeffizienten $a_{p} \in L_{\mathrm{loc}}^{2}(\Omega)$ sind so regulär, dass der formal adjungierte Ausdruck

$$
l^{\prime}=\sum_{p \leqq 2 m} a_{p}^{*} D^{p}
$$

mit $a_{p}^{*} \in L_{\mathrm{loc}}^{2}(\Omega)$ existiert.

ii) Es existieren zwei reelle Konstanten $c_{0}>0$ und $k_{0}$ derart, dass die in 4.1 erklärten Operatoren $L$ und $L^{\prime} \quad(t=m)$ die Ungleichungen

$$
\operatorname{Re}(u, L u)_{0} \geqq c_{0}\|u\|_{m}^{2}-k_{0}\|u\|_{0}^{2}
$$

und

$$
\operatorname{Re}\left(v, L^{\prime} v\right)_{0} \geqq c_{0}\|v\|_{m}^{2}-k_{0}\|v\|_{0}^{2}
$$

für alle $u \in D(L) \quad$ bzw. $v \in D\left(L^{\prime}\right)$ erfüllen $\left.{ }^{7}\right)$.

Dann gelten die folgenden Aussagen:

a) Falls $\operatorname{Re} \lambda \geqq k_{0}$ ist, hat Problem I für die Differentialgleichung

$$
(l+\lambda) u=f
$$

für jedes $f \in L^{2}(\Omega)$ und jedes zulässige $g \in L^{2}(\Omega)$ genau eine Lösung.

$\left.{ }^{7}\right)$ In dem hier betrachteten allgemeinen Fall folgen diese Ungleichungen nicht aus der gleichmässig starken Elliptizität von $l$. 
b) Ist das Gebiet $\Omega$ beschränkt, so gilt der Fredholmsche Alternativsatz: Die Gleichungen $l u=0$ und $l^{\prime} v=0$ haben dieselbe endliche Anzahl linear unabhängiger schwacher Lösungen mit »homogenen Randdaten»

$$
u_{1}, \ldots, u_{h} \in H_{0}^{m}(\Omega) \quad \text { bzw. } \quad v_{1}, \ldots, v_{h} \in H_{0}^{m}(\Omega) .
$$

Problem I für die Gleichung $l u=f$ hat eine (bis auf eine Linearkombination der Funktionen $u_{1}, \ldots, u_{h}$ eindeutig bestimmte) schwache Lösung dann und nur dann, wenn

$$
\left(v_{j}, f\right)_{0}-\widetilde{b_{g}}\left(v_{j}\right)=0
$$

für $j=1, \ldots, h$ gilt.

4.5. Wir beweisen zunächst die Behauptung a) von Satz 4.3. Dem Differentialausdruck $l$ sei gemäss (1.10) der Operator $T$ des Hilbertraumes $H_{0}^{m}(\Omega)$ zugeordnet. Auf Grund des Satzes von Fréchet und Riesz lässt sich das Skalarprodukt $(u, v)_{0}$ für Funktionen $u, v \in H_{0}^{m}(\Omega)$ in der Form $(S u, v)_{m}$ darstellen, wobei $S$ ein beschränkter selbstadjungierter Operator des Raumes $H_{0}^{m}(\Omega)$ ist. Da

$$
\left(\left(l^{\prime}+\bar{\lambda}\right) \varphi, v\right)_{0}=((T+\bar{\lambda} S) \varphi, v)_{m}
$$

für alle $\varphi \in C_{0}^{\infty}(\Omega), v \in H_{0}^{m}(\Omega)$ gilt, entspricht analog der Definition (1.10) dem Ausdruck $l+\lambda$ der Operator $T+\bar{\lambda} S$ des Raumes $H_{0}^{m}(\Omega)$.

Um für $\operatorname{Re} \lambda \geqq k_{0}$ die Existenz (mindestens) einer Lösung zu zeigen, genügt es nach Satz 1.1, die Beziehung

$$
W\left(T^{*}+\lambda S\right)=H_{0}^{m}(\Omega)
$$

zu beweisen. Auf Grund der Ungleichung (4.9) ist

$$
\|(T+\bar{\lambda} S) \varphi\|_{m} \geqq c_{0}\|\varphi\|_{m}
$$

für alle $\varphi \in C_{0}^{\infty}(\Omega)$; der Operator $T+\bar{\lambda} S$ genügt also wegen seiner Abschliessbarkeit den Voraussetzungen von Lemma 4.1. Dieses Lemma ergibt uns die Relation (4.10).

Die Eindeutigkeit der Lösung ist nach der Beziehung (1.11) der Gleichung

$$
K\left(T^{*}+\lambda S\right)=\{0\}
$$

äquivalent. Wir zeigen, dass (4.12) für $\operatorname{Re} \lambda \geqq k_{0}$ erfüllt ist: Sei $z$ ein beliebiges Element aus dem Kern von $T^{*}+\lambda S$. Dann ist für alle $\varphi \in C_{0}^{\infty}(\Omega)$

$$
\begin{aligned}
0 & =\left(\varphi,\left(T^{*}+\lambda S\right) z\right)_{m}=((T+\bar{\lambda} S) \varphi, z)_{m} \\
& =\left(\left(l_{0}^{\prime}+\bar{\lambda}\right) \varphi, z\right)_{0}=\left(\varphi,\left(\left(l_{0}^{\prime}\right)^{+}+\lambda\right) z\right)_{0} \\
& =\left(\varphi,\left(L_{\max }+\lambda\right) z\right)_{0}=(\varphi,(L+\lambda) z)_{0}
\end{aligned}
$$


es ergibt sich

$$
(L+\lambda) z=0
$$

woraus zusammen mit (4.8) die Behauptung $z=0$ folgt.

4.6. Für den Beweis von Satz 4.3.b) benötigen wir zwei Lemmata, die wir in diesem Abschnitt beweisen. Beim Beweis von Lemma 4.4 benützen wir die schon gezeigte Aussage a) von Satz 4.3.

Lemma 4.4. Der Differentialausdruck $l$ erfülle die Voraussetzungen i) und ii). Ferner sei $T$ der durch (1.10) definierte Operator des Hilbertraumes $H_{0}^{m}(\Omega)$, und $T^{*}$ bezeichne den zu $T$ im Raume $H_{0}^{m}(\Omega)$ adjungierten Operator. Dann gilt $^{8}$ )

$$
\overline{\left(T^{*}\right)_{0}}=T^{*} \text {. }
$$

Beweis. Dem Differentialausdruck $l+k_{0}$ ist analog der Definition (1.10) der Operator $T+k_{0} S$ des Raumes $H_{0}^{m}(\Omega)$ zugeordnet. Den Resultaten aus 4.5 entnehmen wir

$$
K\left(T^{*}+k_{0} S\right)=\{0\} .
$$

Entsprechend ist dem Ausdruck $l^{\prime}+k_{0}$ der Operator $\left(T^{*}\right)_{0}+k_{0} S$ zugeordnet, und man erhält die Beziehung

$$
K\left(\left(\left(T^{*}\right)_{0}\right) *+k_{0} S\right)=\{0\} .
$$

Aus der orthogonalen Zerlegung

$$
H_{0}^{m}(\Omega)=K\left(\left(\left(T^{*}\right)_{0}\right)^{*}+k_{0} S\right) \oplus \overline{W\left(\overline{\left(T^{*}\right)_{0}}+k_{0} S\right)}
$$

des Raumes $H_{0}^{m}(\Omega)$ folgt somit

$$
\overline{W\left(\overline{\left(T^{*}\right)_{0}}+k_{0} S\right)}=H_{0}^{m}(\Omega) \text {. }
$$

Wegen der sich aus (4.8) ergebenden Ungleichung

$$
\left\|\left(\left(T^{*}\right)_{0}+k_{0} S\right) \varphi_{m}^{\prime} \geqq c_{0}\right\| \varphi \|_{m}
$$

für alle $\varphi \in C_{0}^{\infty}(\Omega)$ ist die Wertemenge des Operators $\overline{\left(T^{*}\right)_{0}}+k_{0} S$ abgeschlossen in $H_{0}^{m}(\Omega)$ (vgl. [27], Satz 2, S. 475); es gilt also

$$
W\left(\overline{\left(T^{*}\right)_{0}}+k_{0} S\right)=H_{0}^{m}(\Omega) .
$$

Auf Grund der Inklusion

$$
\overline{\left(T^{*}\right)_{0}}+k_{0} S \subset T^{*}+k_{0} S
$$

ist folglich auch

$\left.{ }^{8}\right)$ Mit $A_{0}$ bezeichnen wir die Einschränkung des Operators $A$ auf die Menge $C_{\mathbf{0}}^{\infty}(\Omega)$ und mit $\bar{A}$ die Abschliessung von $A$ im Raume $H_{0}^{m}(\Omega)$. 


$$
W\left(T^{*}+k_{0} S\right)=H_{0}^{m}(\Omega) .
$$

Die Operatoren $\overline{\left(T^{*}\right)_{0}}+k_{0} S$ und $T^{*}+k_{0} S$ sind wegen (4.13) und (4.15) invertierbar. Da

$$
\left(\overline{\left(T^{*}\right)_{0}}+k_{0} S\right)^{-1} \subset\left(T^{*}+k_{0} S\right)^{-1}
$$

und da die Definitionsmengen der Operatoren $\left(\overline{\left(T^{*}\right)_{0}}+k_{0} S\right)^{-1}$ und $\left(T^{*}+k_{0} S\right)^{-1}$ nach (4.14) und (4.16) zusammenfallen, gilt

$$
\left(\overline{\left(T^{*}\right)_{0}}+k_{0} S\right)^{-1}=\left(T^{*}+k_{0} S\right)^{-1},
$$

und es folgt

$$
\overline{\left(T^{*}\right)_{0}}=T^{*} .
$$

Lemma 4.5. Der Differentialausdruck $l$ erfülle die Voraussetzungen i) und $i i)$. Ferner sei $\mathfrak{\mathfrak { H }}^{+0}$ der in Satz 3.2 definierte Unterraum von $H_{0}^{m}(\Omega)$. Ein Element $v \in H_{0}^{m}(\Omega)$ ist dann und nur dann in $\mathfrak{S}^{+0}$ enthalten, wenn $(v, l \varphi)_{0}=0$ für alle $\varphi \in C_{0}^{\infty}(\Omega)$ gilt.

Beweis. Da $\mathfrak{H}^{+0}=K\left(T^{*} T^{* *}\right)=K\left(T^{* *}\right)$, liegt wegen der orthogonalen Zerlegung

$$
H_{0}^{m}(\Omega)=K\left(T^{* *}\right) \oplus \overline{W\left(T^{*}\right)}
$$

ein Element $v \in H_{0}^{m}(\Omega)$ genau dann in $\mathfrak{S}^{+0}$, wenn

$$
\left(v, T^{*} z\right)_{m}=0
$$

für alle $z \in D\left(T^{*}\right)$ gilt. Auf Grund des Lemmas 4.4 sowie der Inklusion

$$
W\left(\overline{\left(T^{*}\right)_{0}}\right) \subset \overline{W\left(\left(T^{*}\right)_{0}\right)}
$$

ist (4.17) äquivalent der Beziehung

$$
\left(v,\left(T^{*}\right)_{0} \varphi\right)_{m}=(v, l \varphi)_{0}=0
$$

für alle $\varphi \in C_{0}^{\infty}(\Omega)$, w. z. b. w.

4.7. Wir beweisen jetzt die Behauptung b) von Satz $4.3^{9}$ ).

Aus Satz 3.3 folgt unter Berücksichtigung der Lemmata 3.4 und 4.5, dass Problem I für die Gleichung $l u=f$ dann und nur dann lösbar ist, wenn

$$
(v, f)_{0}-\widetilde{b_{g}}(v)=0
$$

für alle schwachen Lösungen $v \in H_{0}^{m}(\Omega)$ der Gleichung $l^{\prime} v=0$ gilt.

9) Dieser Beweis unterscheidet sich vom bekannten Verfahren für den Beweis ron Satz 4.2.b) (vgl. [2], [3], [6]); dort wird nämlich an einigen Stellen die Beschränktheit der Bilinearform $B$ wesentlich verwendet. 
Es bleibt zu zeigen, dass die Gleichungen $l u=0$ und $l^{\prime} v=0$ dieselbe endliche Anzahl $h$ linear unabhängiger schwacher Lösungen $u_{1}, \ldots, u_{h} \in H_{0}^{m}(\Omega) \quad$ bzw. $v_{1}, \ldots, v_{h} \in H_{0}^{m}(\Omega)$ besitzen.

Nach Satz 4.3.a) existiert für eine beliebig gewählte Funktion $f \in L^{2}(\Omega)$ genau eine Lösung $u \in H_{0}^{m}(\Omega)$ von Problem I für die Gleichung $\left(l+k_{0}\right) u=f$ mit $g=0$; es gibt also eine und nur eine Funktion $u \in H_{0}^{m}(\Omega)$, die der Gleichung

$$
\left(\left(l^{\prime}+k_{0}\right) \varphi, u\right)_{0}=(\varphi, f)_{0}
$$

für alle $\varphi \in C_{0}^{\infty}(\Omega)$ genügt. Nach Definition des Operators $L$ ist (4.18) der Gleichung

$$
u=\left(L+k_{0}\right)^{-1} f
$$

äquivalent.

Eine Funktion $u \in H_{0}^{m}(\Omega)$ ist schwache Lösung der Gleichung $l u=f$ dann und nur dann, wenn sie die Gleichung

$$
\left(\left(l^{\prime}+k_{0}\right) \varphi, u\right)_{0}=\left(\psi, k_{0} u+f\right)_{0}
$$

für alle $\varphi \in C_{0}^{\infty}(\Omega)$ erfüllt, d. h. wenn

$$
u=\left(L+k_{0}\right)^{-1}\left(k_{0} u+f\right)
$$

gilt. Wir führen die Bezeichnungen

$$
A=k_{0}\left(L+k_{0}\right)^{-1}, \quad f^{\prime}=\left(L+k_{0}\right)^{-1} f
$$

ein. Der infolge (4.8) beschränkte Operator $A$ bildet $L^{2}(\Omega)$ in $H_{0}^{m}(\Omega)$ ab; der Operator $A$ ist folglich, als Abbildung von $L^{2}(\Omega)$ in sich betrachtet, kompakt.

Somit ist eine Funktion $u \in L^{2}(\Omega)$ genau dann in $H_{0}^{m}(\Omega)$ enthalten und schwache Lösung der Gleichung $l u=f$, wenn

$$
u-A u=f^{\prime}
$$

gilt.

Nach den Fredholmschen Sätzen über Gleichungen mit kompakten Operatoren ist die Wertemenge $W(I-A)$ des Operators $I-A$ in $L^{2}(\Omega)$ abgeschlossen, und die Dimensionen des Kerns $K(I-A)$ und des Orthogonalkomplementes $L^{2}(\Omega) \ominus W(I-A)$ von $W(I-A)$ sind endlich und gleich (vgl. z. B. [27], S. 363-365).

Es bezeichne $k$ die maximale Anzahl linear unabhängiger schwacher Lösungen $u \in H_{0}^{m}(\Omega)$ der Gleichung $l u=0$, und die maximale Anzahl linęs unabhängiger schwacher Lösungen $v \in H_{0}^{m}(\Omega)$ der Gleichung $l^{\prime} v=0$ sei $h$. Aus dem eben Erwähnten folgt $k<\infty$. Wir zeigen 
indirekt, dass $h \leqq k$ gilt. Unter der Annahme, dass $h>k$ ist, gibt es $k+1$ linear unabhängige schwache Lösungen $v_{1}, \ldots, v_{k+1} \in H_{0}^{m}(\Omega)$ der Gleichung $l^{\prime} v=0$. Auf Grund des bereits bewiesenen Teils der Aussage b) von Satz 4.3 ist Problem I mit $g=0$ für die Gleichung $l u=f$ für keine Funktion $f \neq 0$ aus der von $v_{1}, \ldots, v_{k+1}$ aufgespannten linearen Menge $M$ lösbar. Deshalb ist auch das dazu äquivalente Problem

$$
u-A u=f^{\prime}
$$

für keine Funktion $f^{\prime} \neq 0$ aus der von den Vektoren $v_{j}^{\prime}=\left(L+k_{0}\right)^{-1} v_{j}$ aufgespannten linearen Menge $M^{\prime}$ lösbar. Weil die lineare Unabhängigkeit bei der eineindeutigen Abbildung $\left(L+k_{0}\right)^{-1}$ erhalten bleibt, ist $\operatorname{dim} M^{\prime}=k+1$.

Der Raum $L^{2}(\Omega)$ ist als direkte Summe

$$
L^{2}(\Omega)=W(I-A) \dot{+} M^{\prime} \dot{+} X
$$

darstellbar, wobei $X$ ein linearer Raum ist. Das Orthogonalkomplement $L^{2}(\Omega) \ominus W(I-A)$ von $W(I-A)$ hat folglich die Dimension

$$
\operatorname{dim}\left(M^{\prime}+X\right)=k+1+\operatorname{dim} X \geqq k+1 .
$$

Es existieren somit (mindestens) $k+1$ linear unabhängige Elemente $u_{i} \in L^{2}(\Omega) \quad(i=1, \ldots, k+1) \quad$ mit der Eigenschaft

$$
u_{i}-A u_{i}=0 .
$$

Es ist $u_{i}=A u_{i} \in H_{0}^{m}(\Omega)$. Die Gleichung $l u=0$ besitzt also $k+1$ linear unabhängige schwache Lösungen $u_{i} \in H_{0}^{m}(\Omega)$, was im Widerspruch zur Definition von $k$ steht. Wir haben die Beziehung $h \leqq k<\infty$ bewiesen. Die symmetrische Betrachtung ergibt $k \leqq h$.

4.8. Wir haben die Fragen der Regularität der schwachen Lösungen hier nicht behandelt. Für elliptische Differentialgleichungen mit beschränkten Koeffizienten sind diese Fragen ausführlich studiert worden. Da es sich bei der Untersuchung der inneren Regularität einer Lösungsfunktion um lokale Eigenschaften handelt, können diese Resultate direkt in unsere Theorie übernommen werden. Schwieriger ist die Diskussion des Randverhaltens einer Lösung; diese Frage ist für den Fall, dass sich die Koeffizienten des Differentialausdruckes in der Umgebung des Randes des Definitionsgebietes nicht regulär verhalten, in der Literatur bisher kaum systematisch betrachtet worden.

4.9. Das abschliessende Beispiel zeigt einen Differentialausdruck, der den Voraussetzungen von Satz 4.3 genügt, ohne die Bedingungen von Satz $4.2 \mathrm{zu}$ erfüllen. 
Beispiel 4.6. Der Ausdruck

$$
l=-\Delta+q
$$

erfülle die folgenden Voraussetzungen:

1) Für alle $x \in \Omega$ gilt die Ungleichung $\operatorname{Re} q(x) \geqq k$, und die Funktion $\operatorname{Re} q$ wächst gegen den Rand von $\Omega$ derart an, dass der durch den Ausdruck

$$
l_{\mathrm{Re}}=-\Delta+\operatorname{Re} q
$$

auf der Menge $C_{0}^{\infty}(\Omega)$ erzeugte Differentialoperator $\left(l_{\mathrm{Re}}\right)_{0}$ in $L^{2}(\Omega)$ wesentlich selbstadjungiert ist (vgl. [10]).

2) Die Funktion $\operatorname{Im} q$ ist beschränkt in $\Omega$.

Dann erfüllt der Ausdruck $l$ die Voraussetzungen von Satz 4.3.

Beweis. Für alle $\varphi \in C_{0}^{\infty}(\Omega)$ gilt

$$
\operatorname{Re}(\varphi, l \varphi)_{0}=\operatorname{Re}\left(\varphi, l^{\prime} \varphi\right)_{0} \geqq\|\varphi\|_{1}^{2}-(1-k)\|\varphi\|_{0}^{2} .
$$

Da der Operator $A=\operatorname{Im} q$ beschränkt ist in $L^{2}(\Omega)$, ergibt sich

$$
L_{\min }=L_{\max }=L \quad \text { bzw. } \quad L_{\min }^{\prime}=L_{\max }^{\prime}=L^{\prime} ;
$$

aus (4.20) folgen durch Abschliessung die Ungleichungen (4.8) bzw. (4.9).

Der Ausdruck $l$ genügt unter diesen Voraussetzungen nicht den Bedingungen von Satz 4.2, denn die Funktion $q$ ist nicht beschränkt in $\Omega$.

\section{Zusatz bei der Korrektur}

(November 1968)

Mit der in Kapitel 4 angewandten Methode lässt sich die nachstehende Verschärfung von Satz 4.3 beweisen (vgl. Lemma 4.4):

Satz. Es bezeichne $\Omega$ ein beschränktes Gebiet in $R^{n}$, und es seien

$$
\begin{aligned}
l & =\sum_{|p| \leqq 2 m} a_{p} D^{p} \quad\left(a_{p} \in L_{\mathrm{loc}}^{2}(\Omega)\right), \\
l^{\prime} & =\sum_{|p| \leqq 2 m} a_{p}^{*} D^{p} \quad\left(a_{p}^{*} \in L_{\mathrm{loc}}^{2}(\Omega)\right)
\end{aligned}
$$

zwei zueinander adjungierte Differentialausdrücke der Ordnung $2 \mathrm{~m}$. Die Gårdingsche Ungleichung

$$
\operatorname{Re}(\varphi, l \varphi)_{0} \geqq c_{0}\|\varphi\|_{m}^{2}-k_{0}\|\varphi\|_{0}^{2} \quad\left(c_{0}>0\right)
$$

sei für alle $\varphi \in C_{0}^{\infty}(\Omega)$ erfüllt. Es bezeichne $T$ den gemäss (1.10) dem Ausdruck $l$ zugeordneten Operator des Raumes $H_{0}^{m}(\Omega)$. Dann ist die Gültigkeit der Beziehung 


$$
\overline{\left(T^{*}\right)_{0}}=T^{*}
$$

notwendig und hinreichend dafür, dass für $l$ der Fredholmsche Alternativsatz gilt, d.h. dass die folgenden zwei Aussagen gelten:

a) Falls $\operatorname{Re} \lambda \geqq k_{0}$, besitzt Problem I mit $t=m$ für die Gleichung $(l+\lambda) u=f$ für jedes $f \in L^{2}(\Omega)$ und für jedes zulässige $g \in L^{2}(\Omega)$ genau eine Lösung.

b) Die Gleichungen $(l+\lambda) u=0$ und $\left(l^{\prime}+\bar{\lambda}\right) v=0$ haben dieselbe endliche Anzahl linear unabhängiger schwacher Lösungen mit »homogenen Randdaten» $u_{1}, \ldots, u_{h} \in H_{0}^{m}(\Omega)$ bzw. $v_{1}, \ldots, v_{h} \in H_{0}^{m}(\Omega)$. Problem I für die Gleichung $(l+\lambda) u=f$ hat eine (bis auf eine Linearkombination der Funktionen $u_{1}, \ldots, u_{h}$ eindeutig bestimmte) Lösung dann und nur dann, wenn

$$
\left(v_{j}, f\right)_{0}-\widetilde{b_{g}}\left(v_{j}\right)-\bar{\lambda}\left(v_{j}, g\right)_{0}=0
$$

für $j=1, \ldots, h$ gilt.

Man bemerke, dass die Gültigkeit der Voraussetzungen von Satz 4.2 die Beschränktheit des Operators $T$ impliziert und dass (Z.2) dann trivialerweise erfüllt ist. Ein weiteres Beispiel einer hinreichenden Bedingung für die Richtigkeit von (Z.2) stellt das Bestehen der Ungleichungen (4.8) und (4.9) dar.

Eidgenössische Technische Hochschule

Zürich, Schweiz 


\section{Literatur}

[1] Achieser, N. I., und I. M. Glasmann: Theorie der linearen Operatoren im Hilbert-Raum. - [Dritte Auflage.] Mathematische Lehrbücher und Monographien I. IV, Akademie-Verlag, Berlin, 1960.

[2] Agmon, S.: Lectures on elliptic boundary value problems. - Van Nostrand mathematical studies 2, D. Van Nostrand Company, Inc., Princeton (N.J.) / Toronto / New York / London, 1965.

[3] Bers, L., und M. Schechter: Elliptic equations. - Im Werke: Partial differential equations. Lectures in applied mathematics III, Interscience Publishers, a division of John Wiley \& Sons, Inc., New York / London / Sydney, 1964, S. 131-299.

[4] Browder, F. E.: A remark on the Dirichlet problem for non-elliptic selfadjoint partial differential operators. - Rend. Circ. Mat. Palermo (II) 6, 1957 , S. $249-253$.

[5] -»- On the Dirichlet problem for linear non-elliptic partial differential equations, II. - Rend. Circ. Mat. Palermo (II) 7, 1958, S. 303-308.

[6] Friedman, A.: Partial differential equations of parabolic type. - PrenticeHall, Inc., Englewood Cliffs (N.J.), 1964.

[7] Ginzburg, YU. P., und I. S. Ioknvidov: The geometry of inf inite-dimensional spaces with a bilinear metric. - Russian Math. Surveys 17:4, 1962, S. $1-51$.

[8] Hildebrandt, S.: Rand- und Eigenwertaufgaben bei stark elliptischen Systemen linearer Differentialgleichungen. - Math. Ann. 148, 1962, S. 411-429.

[9] Hildebrandt, S., und E. Wienholtz: Constructive proofs of representation theorems in separable Hilbert space. - Comm. Pure Appl. Math. 17, 1964 , S. $369-373$.

[10] Jörgens, K.: Wesentliche Selbstadjungiertheit singulärer elliptischer Differentialoperatoren zweiter Ordnung in $C_{0}^{\infty}(G)$. - Math. Scand. 15, 1964, S. $5-17$.

[11] Lax, P. D., und A. N. Milgram: Parabolic equations. - Im Werke: Contributions to the theory of partial differential equations. Annals of mathematics studies 33, Princeton University Press, Princeton (N.J.), 1954, S. $167-190$.

[12] Lions, J. L.: Equations differentielles operationnelles et problèmes aux limites. - Die Grundlehren der mathematischen Wissenschaften 111, Berlin / Göttingen / Heidelberg, 1961.

[13] Littunan, W.: Remarks on the Dirichlet problem for general linear partial differential equations. - Comm. Pure Appl. Math. 11, 1958, S. 145-151.

[14] LouhivaARA, I. S.: Über das erste Randwertproblem für die Differentialgleichung $u_{x x}+u_{y y}+q u+f=0$. - Ann. Acad. Sci. Fennicæ A. I. 183,1954 . 
[15] LouhivaARA, I. S.: Über das zweite und dritte Randwertproblem für die Differentialgleichung $u_{x x}+u_{y y}+q u+f=0$. - Ann. Acad. Sci. Fennicæ A. I. 203, 1955. Auch im Werke: Commentationes in honorem Rolf Herman Nevanlinna die natali eius sexagesimo ediderunt amici et dispuli. Helsinki, 1955.

[16] - -- Bemerkung zur Theorie der Nevanlinnaschen Räume. - Ann. Acad. Sci. Fennicæ A. I. 232, 1956.

[17] - - Über das Dirichletsche Problem für die selbstadjungierten linearen partiellen Differentialgleichungen zweiter Ordnung. - Rend. Circ. Mat. Palermo (II) 5, 1956, S. 260-274.

[18] -»- Zur Theorie der Unterräume in linearen Räumen mit indefiniter Metrik. Ann. Acad. Sci. Fennicæ A. I. 252, 1958.

[19] - - Über die neuere Entwicklung der Theorie der linearen Räume mit indefiniten Bilinearformen. - Im Werke: Festband zum 70. Geburtstag von Rolf Nevanlinna, Vorträge, gehalten anlässlich des Zweiten Rolf Nevanlinna -Kolloquiums in Zürich vom 4.-6. November 1965. SpringerVerlag, Berlin / Heidelberg / New York, 1966, S. 66-81.

[20] von NeumanN, J.: Über adjungierte Funktionaloperatoren. - Ann. of Math. (II) 33, 1932, S. 294-310. Auch im Werke: John von Neunans: Collected works. II. Operators, ergodic theory and almost periodic functions in a group. Pergamon Press, Oxford / London / New York/Paris, 1961, S. $242-258$.

[21] Neumark, M. A.: Lineare Differentialoperatoren. - [Zweite Auflage.] Mathematische Lehrbücher und Monographien II. XI, Akademie-Verlag, Berlin, 1963.

[22] Nevanlinna, R.: Über metrische lineare Räume. II. Bilinearformen und Stetigkeit. - Ann. Acad. Sci. Fennicæ A. I. 113, 1952.

[23] - - Über metrische lineare Räume. III. Theorie der Orthogonalsysteme. Ann. Acad. Sci. Fennicæ A. I. 115, 1952.

[24] - - Erweiterung der Theorie des Hilbertschen Raumes. - Medd. Lunds Univ. Mat. Sem. Supplementband tillägnat Marcel Riesz, Tome supplémentaire dédié à Marcel Riesz, 1952, S. 160-168.

[25] - - Über metrische lineare Räume. IV. Zur Theorie der Unterräume. - Ann. Acad. Sci. Fennicæ A. I. 163, 1954.

[26] - - Über metrische lineare Räume. V. Relationen zwischen verschiedenen Metriken. - Ann. Acad. Sci. Fennicæ A. I. 222, 1956.

[27] Smirnow, W. I.: Lehrgang der höheren Mathematik. V. - Hochschulbücher für Mathematik 6, VEB Deutscher Verlag der Wissenschaften, Berlin, 1962.

[28] Stenholm, S.: On linear equations in a Hilbert space. - Ann. Acad. Sci. Fennicæ A. I. 370,1965 . 